\title{
Super-poissonian photon statistics and correlations between pump and probe fields in Electromagnetically Induced Transparency
}

\author{
C. L. Garrido Alzar, L. S. Cruz, J. G. Aguirre Gómez, M. França Santos, I and P. Nussenzveig \\ Instituto de Física, Universidade de São Paulo, Caixa Postal 66318, CEP 05315-970, São Paulo, SP, Brazil.
}

(November 1, 2018)

\begin{abstract}
We have measured the photon statistics of pump and probe beams after interaction with Rb atoms in a situation of Electromagnetically Induced Transparency. Both fields present super-poissonian statistics and their intensities become correlated, in good qualitative agreement with theoretical predictions in which both fields are treated quantum-mechanically. The intensity correlations measured are a first step towards the observation of entanglement between the fields.
\end{abstract}

PACS numbers: 42.50.-p, 42.50.Ar, 42.50.Ct, 42.50.Gy

Electromagnetically Induced Transparency (EIT) [1] is an interference effect that can be observed when threelevel atoms interact simultaneously with two lasers. The recent interest in these systems has been driven by observations of very slow light pulse propagation in EIT media [2, 迎 and even of light storage [5, 6]. Even though it is normally viewed as a quantum interference effect, EIT has a very simple classical counterpart [7,8]. The question of whether there exist intrinsic quantum effects (with no classical analog) in EIT naturally arises in this context. It is even more important in connection with suggested applications in the field of quantum information [9, 10]. Quantum field properties in EIT have been theoretically investigated. Phase-noise squeezing was predicted by Fleischhauer and co-workers [11]. Agarwal [12] found matched photon-statistics for two classical and two quantum fields interacting with three-level atoms, in a situation of Coherent Population Trapping. Jain [13 extended this work, and predicted excess-noise correlations in EIT. In all the above [9 13], at least one of the fields was treated classically. As we will see below, it is our belief that such an approximation is not valid in the EIT situation.

In this Letter we present the first experimental investigation (to our knowledge) of field fluctuations and correlations in EIT. We have performed photon statistics measurements of both pump and probe fields, as a function of the probe field detuning. As predicted by our theoretical treatment, both fields, treated quantummechanically, have super-poissonian statistics. Furthermore, they are coupled by their interaction with the atoms and thus their intensities become correlated.

We begin by describing our theoretical model for threelevel atoms in the $\Lambda$-configuration (ground states $|1\rangle$ and $|2\rangle$, and excited state $|0\rangle$ ) interacting with two quantum modes of the electromagnetic field. In order to simulate the interaction of the atoms with two propagating fields, we use the input-output formalism 14, 15], and consider the interaction between the two fields and the atoms in a ring cavity [16], with external input fields.
For the intracavity field operators we used plane wave and quasi-monochromatic approximations. The interaction hamiltonian is obtained with the usual dipole and rotating-wave approximations

$$
\hat{H}_{\mathrm{int}}=\hbar g_{1} \hat{S}_{1}^{+}(t) \hat{A}_{1}(t)+\hbar g_{2} \hat{S}_{2}^{+}(t) \hat{A}_{2}(t)+\text { h.c. },
$$

where $\hat{A}_{1}\left(\hat{A}_{2}\right)$ is the annihilation operator for intracavity field 1 - pump (intracavity field 2 - probe), $g_{1}\left(g_{2}\right)$ is the atom - field 1 (field 2) coupling strength, and $\hat{S}_{1}^{+}$ $\left(\hat{S}_{2}^{+}\right)$the atomic polarization on the transition $|1\rangle \leftrightarrow|0\rangle$ $(|2\rangle \leftrightarrow|0\rangle)$.

From the Heisenberg equations of motion, we derive quantum Langevin equations 17] for the system operators. In a matrix form, we find [18]

$$
\frac{d \widehat{\bar{X}}(t)}{d t}=-\hat{\mathbf{A}}(t) \hat{\bar{X}}(t)+\mathbf{D} \widehat{\overline{\mathcal{F}}}(t) \text {. }
$$

The vector operator $\hat{\bar{X}}(t)$ has as its components the operators giving the atomic inversions and polarizations (and their hermitian conjugates) corresponding to the two transitions, the coherences between the two ground states and the annihilation and creation operators of both fields. The vector of the Langevin forces acting on the system is given by $\widehat{\overline{\mathcal{F}}}(t)$, and $\mathbf{D}$ is the diffusion matrix. The matrix $\hat{\mathbf{A}}(t)$, in the steady state, will represent the drift matrix. Eq. (2) is a compact form of writing twelve coupled differential equations for the operators.

We are interested in studying the fluctuations of the field operators. This is done by linearizing the operators in Eq. (2) around their stationary values. A new set of twelve coupled differential equations is obtained. It is interesting to examine the equations for the fluctuations of one field and for the corresponding atomic polarization:

$$
\begin{aligned}
\frac{d \delta \hat{A}_{1}}{d t}= & -\left(\frac{\gamma}{2}+i \Delta_{c 1}\right) \delta \hat{A}_{1}-i \frac{g_{1}}{\tau} \delta \hat{S}_{1}^{-}+\sqrt{\frac{\gamma}{\tau}} \delta \hat{A}_{1 i n} \\
\frac{d \delta \hat{S}_{1}^{-}}{d t}= & -\left(\frac{\Gamma_{1}}{4}-i \delta_{L 1}\right) \delta \hat{S}_{1}^{-}+i g_{1} w_{1} \delta \hat{A}_{1}+i g_{1} \alpha_{1} \delta \hat{W}_{1} \\
& -i g_{2} s_{12}^{*} \delta \hat{A}_{2}-i g_{2} \alpha_{2} \delta \hat{S}_{12}^{+}+\hat{F}_{S 1}
\end{aligned}
$$


Here we define $\gamma$ as the cavity linewidth, $\Delta_{c 1}$ cavity detuning for field $1, \tau$ cavity length divided by the speed of light, $\hat{A}_{1 \text { in }}$ the annihilation operator for the input field 1 , $\Gamma_{1}$ spontaneous emission rate from $|0\rangle \rightarrow|1\rangle, \delta_{L 1}$ detuning between field 1 and the corresponding atomic transition, $w_{1}$ steady-state inversion between states $|0\rangle$ and $|1\rangle, \alpha_{1}\left(\alpha_{2}\right)$ steady-state amplitude of field 1 (field 2), $\hat{W}_{1}$ inversion (operator) between states $|0\rangle$ and $|1\rangle, s_{12}^{*}$ steady-state coherence between ground states $|1\rangle$ and $|2\rangle$, $\hat{S}_{12}^{+}$coherence operator, $\hat{F}_{S 1}$ Langevin fluctuation force. The notation $\delta \hat{S}_{1}^{-}$means fluctuations of the corresponding operator.

It is clear from Eqs. (3) and (41) that the fluctuations in field 1 are not only determined by input field fluctuations but also by atomic fluctuations and by fluctuations in field 2. Moreover, in Eq. (位) we notice that noise correlations between the fields will arise, conditioned to the existence of a coherence between the ground states, $s_{12}^{*}$, only important on EIT resonance.

The terms $i g_{1} w_{1} \delta \hat{A}_{1}, i g_{1} \alpha_{1} \delta \hat{W}_{1}, i g_{2} s_{12}^{*} \delta \hat{A}_{2}, i g_{2} \alpha_{2} \delta \hat{S}_{12}^{+}$ in Eq. (四) are all of the same order. This means that we can not neglect the fluctuations of either field with respect to its average (steady-state value). It is not valid to treat one field as classical and the other as quantized. This conclusion also applies to the work by Agarwal [12] and Jain [13] who consider four independent fields (two classical and two quantized) interacting with coherent population trapped (CPT) atoms. The fields used to produce CPT also have inherent quantum fluctuations, which lead to atomic fluctuations and, therefore, should not be neglected.

From the twelve coupled differential equations for the fluctuations, we calculate the spectral matrix $\mathbf{S}(\Omega)$ [19,20] for the noise, which yields the fluctuations of the output cavity fields, after Fourier transform of the input-output relations.

The use of a cavity to simulate an EIT experiment with cw propagating fields (no cavity) is adequate as long as we restrict ourselves to fluctuations around the steady state and do not investigate transient effects. We must also consider the cavity large enough so that the atoms experience neither significant changes in their spontaneous emission rates nor collective effects.

The noise spectra calculated for probe and pump amplitude quadratures and their correlations are plotted in Fig. 1, as a function of the probe frequency. These spectra were calculated for an analysis frequency $\Omega=\Gamma / 6 \pi$ (consistent with the experiment), where $\Gamma$ is the total spontaneous emission rate from the excited state. We took equal coupling constants for both transitions, pump strictly on resonance, and a ratio of intensities $\left[\left|\alpha_{1} / \alpha_{2}\right|^{2}=9\right.$ in Fig. 1 (a)] such that there is a very narrow and deep EIT resonance. For the correlations in Fig. 1 (b), both fields have equal intensities. The number of atoms was of the order of $10^{4}$, which is sufficiently large to justify the linearization method used.

We find super-poissonian statistics for both fields, and intensity correlations peaked at the EIT resonance. Physically we understand this behavior as follows. The coupling of the fields with the atoms introduces a spreading in the photon distributions associated to each field. The atoms act as "beamsplitters", redistributing photons between both modes, in such a way that the mean numbers do not vary appreciably but the variances are modified. Taking absorption into account, we expect this effect to be reduced, since absorption introduces randomness in the photon redistribution process. We therefore expect to observe a maximum effect for minimum absorption, which corresponds to the EIT resonance. The predicted correlations also depend on the presence of absorption. In the EIT situation, the transmission of one field depends on the (fluctuating) intensity of the other field (cross Kerr effect), leading to intensity correlations. The experimental results presented below are in very good qualitative agreement with these predictions.
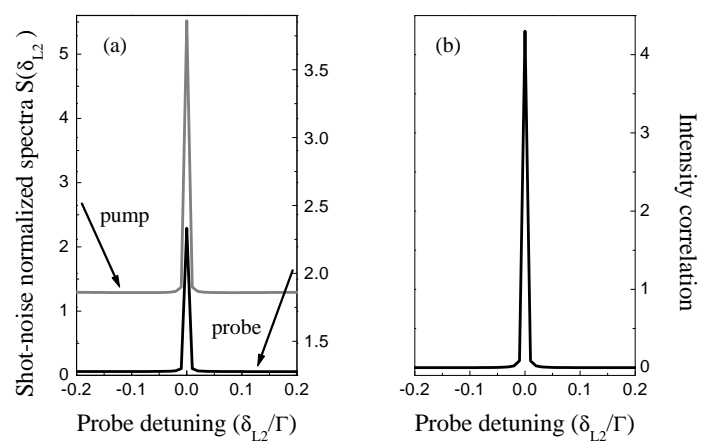

FIG. 1. Theoretical predictions. (a) Super-poissonian photon statistics for both fields, as a function of probe detuning. The left vertical axis is for the pump (gray) and right axis for the probe (black). (b) Intensity correlations as a function of probe detuning (in units of $\Gamma$ ).

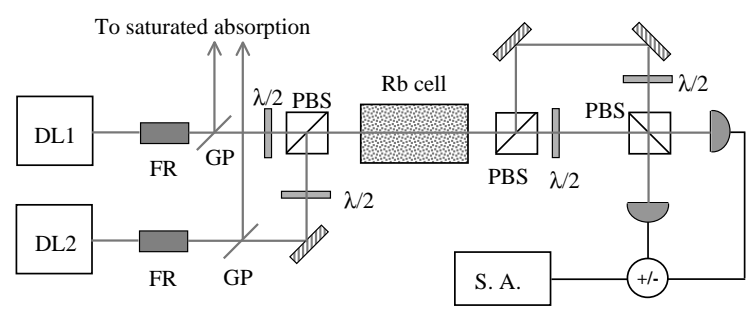

FIG. 2. Sketch of the experimental setup. DL1 and DL2: extended-cavity diode lasers; FR: Faraday rotator; GP: glass plate; PBS: polarizing beamsplitter cube; S.A.: spectrum analyzer.

Our experimental setup is sketched in Fig. 2. Measurements were performed in a $5 \mathrm{~cm}$ long room-temperature $\mathrm{Rb}$ vapor cell. Two independent extended-cavity diode lasers (ECDLs) provide the pump and probe fields. A 
small portion of each beam is extracted and sent to auxiliary saturated absorption cells, used as frequency references. The pump beam is tuned to the ${ }^{85} \mathrm{Rb} \mid 5 S_{1 / 2}, F=$ $3\rangle \rightarrow\left|5 P_{3 / 2}, F^{\prime}=3\right\rangle$ transition, while the probe beam is scanned across the Doppler-broadened $\left|5 S_{1 / 2}, F=2\right\rangle \rightarrow$ $\mid 5 P_{3 / 2}, F^{\prime}=1,2$, or 3$\rangle$ line. The two beams have orthogonal polarizations and are combined by means of a polarizing beamsplitter cube (PBS). At the output of the cell we can separate the beams again with another PBS. We then have the option of detecting either beam with a balanced detection setup 21. Half-wave plates also enable us to recombine the two fields and measure the photon statistics of the sum and difference intensities of the two beams. From these we can extract the intensity correlations.

The usual EIT signals are observed by sending only the probe beam into the detection region and measuring the DC (average) intensity. The intensity transmitted through the cell has a narrow peak as a function of the probe frequency. The width is much narrower than the Doppler width, which is a signature of the interference effect. Next, we measure the photon statistics (intensity fluctuations) for the probe beam, yielding the signal presented in Fig. 3 (a). For the range of frequencies spanned, the photon statistics is always super-poissonian, but it presents a sharp peak (almost $20 \mathrm{~dB}$ ) corresponding to the EIT resonance. In this measurement, the initial photon statistics of the probe beam is poissonian (for the frequency of analysis chosen, the pump initially has super-poissonian statistics, but the theory predicts this effect even when both fields have poissonian statistics). The pump photon statistics presents similar behavior, as seen in Fig. 3 (b). In Fig. 3 (c) and (d) we present the corresponding Fano factors for both probe and pump fields, respectively. The Fano factor is given by the ratio of the intensity fluctuations to the average intensity (shot noise level), and we plot it on a linear scale. In these measurements, the probe and pump intensities were $14.5 \mathrm{~mW} / \mathrm{cm}^{2}$ and $63.8 \mathrm{~mW} / \mathrm{cm}^{2}$, respectively. The observed behavior agrees very well with the theoretical predictions of Fig. 1.

The noise spectra were recorded using a spectrum analyzer (HP $8560 \mathrm{E})$ in the zero span mode $(R B W=$ $300 \mathrm{kHz}, V B W=3 \mathrm{kHz}$ ), with a center (analysis) frequency of $2 \mathrm{MHz}$. This frequency is in a window such that the probe laser has poissonian photon statistics and the electronic noise is safely below shot noise (more than $5 \mathrm{~dB}$ ). The pump laser's noise is $\sim 8 \mathrm{~dB}$ above shot noise (and shows little variation with the analysis frequency). The analyzer is triggered by the same signal used to scan the probe frequency. The photodetectors are EG\&G FND-100 with a nominal efficiency of $\sim 70 \%$. We checked that none of the measurements is influenced by saturation in the detection. This is done by introducing neutral density filters before detection and observing that the photon statistics becomes poissonian, with a lin- ear dependence on absorption.

For typical EIT signals, in the presence of a strong pump field, the shot noise level also peaks, as expected, following the increasing DC intensity on EIT resonance. However, if we lower the pump power, this effect tends to disappear, since incoherent effects then dominate over the coherent pumping on the transition $\left|5 S_{1 / 2}, F=3\right\rangle \rightarrow$ $\left|5 P_{3 / 2}, F^{\prime}=3\right\rangle$. The probe DC signal, and consequently the shot noise level, no longer show any evidence of a coherent effect in the atomic medium for pump intensities lower than $0.45 \mathrm{~mW} / \mathrm{cm}^{2}$, for a probe intensity of $13.3 \mathrm{~mW} / \mathrm{cm}^{2}$ (it is the ratio of pump to probe intensities that is relevant). On the other hand, by looking at the photon statistics, the coherent effect is still clearly identifiable.

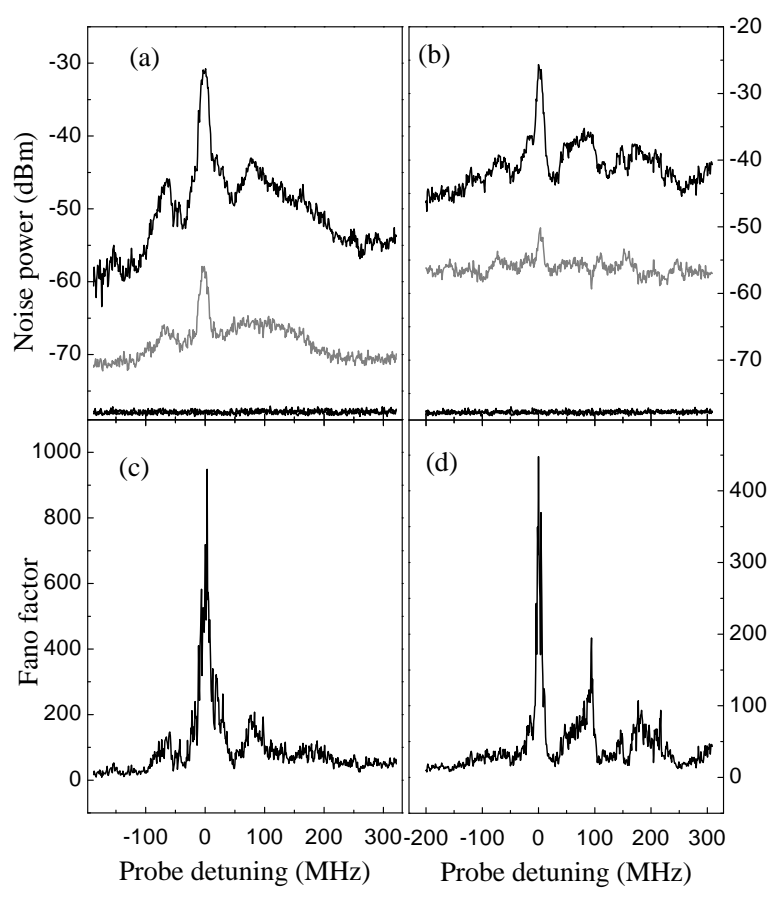

FIG. 3. (a) and (b) Noise spectra of the probe and pump beams, respectively, as a function of the probe frequency and corresponding shot (in gray) and electronic noise (lower traces). (c) and (d) Fano factors deduced from (a) and (b).

We have also measured the noise properties of the sum and difference beam intensities. This is done by sending each beam to one of the detectors. By subtracting the intensity-difference noise from the intensity-sum, we observe a clear correlation on the EIT resonance. The corresponding shot-noise level is measured by mixing the two beams so that each detector receives half of each beam. The results are presented in Fig. 4. We observe $\sim 7 \mathrm{~dB}$ splitting between the sum and difference fluctuations. For photon statistics, this corresponds to a correlation term $2 C=4\left\{\left\langle n_{1} n_{2}\right\rangle-\left\langle n_{1}\right\rangle\left\langle n_{2}\right\rangle\right\}$. 


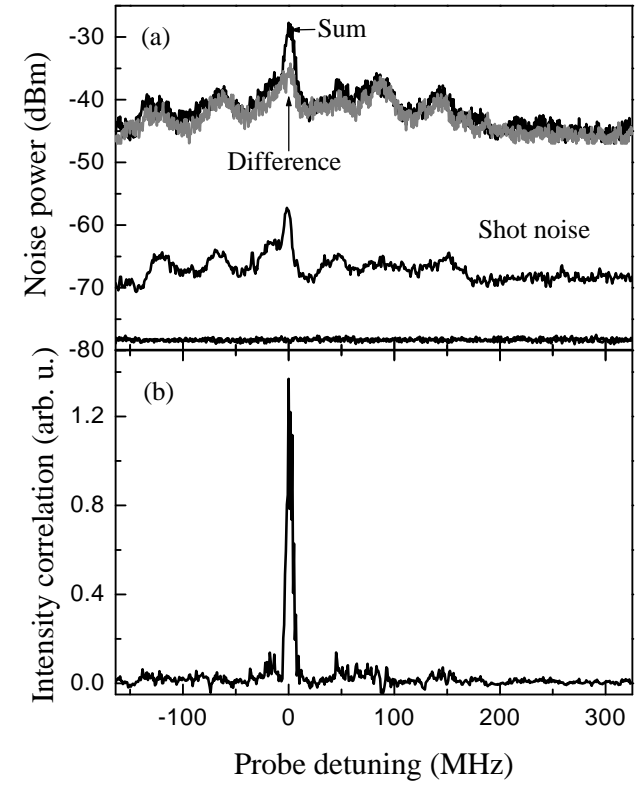

FIG. 4. (a) Measurements of the noise in the sum (black) and difference (gray) intensities of pump and probe beams. The difference observed on EIT resonance is a measurement of the intensity correlation (b), on a linear scale, created between both fields. Pump and probe intensities: $7.8 \mathrm{~mW} / \mathrm{cm}^{2}$ and $7.5 \mathrm{~mW} / \mathrm{cm}^{2}$, respectively.

In summary, we have measured the photon statistics of probe and pump beams after interaction with an atomic medium, in a situation of Electromagnetically Induced Transparency. We observe super-poissonian statistics, peaked on EIT resonance. We have also observed intensity correlations between the two initially independent fields, as a result of their interaction with the atoms. The experimental result features agree very well with our theoretical predictions. The balanced detection scheme leads to enhanced sensitivity for detecting coherent effects in the atomic medium. The importance of the correlations predicted and observed is two-fold. On the one hand, this is a very strong indication that, when investigating quantum properties of one of the fields in a coherent situation (such as EIT), one can not treat either field as classical, as done by several authors. The coherent exchange of photons between the fields, which is at the very origin of the EIT effect, introduces correlations between their fluctuations. Another important issue is the nature of the correlations between both fields. Quantum correlations (entanglement) between intense fields can be used in the context of quantum information. This would be the dual of the experiment by Julsgaard and co-workers 22], in which correlations between atoms in two different vapor cells were created using light. In the present experiment, we have made the first step in this direction by demonstrating the existence of correlations. However, in order to distinguish between classical and quantum correlations, we will need an improved exper- imental setup. A new calculation shows that entanglement between pump and probe fields can be produced in the EIT situation, and its observation will require measurements of quadrature fluctuations of both fields [23].

We thank Profs. L. M. Narducci and H. M. Nussenzveig for critically reading the manuscript, and S. Simionatto for technical assistance. One of us (M.F.S.) would like to thank Prof. S. Salinas for his hospitality in São Paulo. This research was funded by FAPESP. Additional support was provided by the Brazilian agencies CAPES and $\mathrm{CNPq}$.

* Present address: Blackett Laboratory, Imperial College, London SW7 2BW, United Kingdom.

[1] See, for example, S. E. Harris, Phys. Today 50 (7), 36 (1997).

[2] L. V. Hau et al., Nature (London) 397, 594 (1999).

[3] M. M. Kash et al., Phys. Rev. Lett. 82, 5229 (1999).

[4] D. Budker et al., Phys. Rev. Lett. 83, 1767 (1999).

[5] C. Liu et al., Nature (London) 409, 490 (2001).

[6] D. F. Phillips et al., Phys. Rev. Lett. 86, 783 (2001).

[7] C. L. Garrido Alzar, M. A. G. Martinez, and P. Nussenzveig, Am. J. Phys. 70, 37 (2002).

[8] P. R. Hemmer and M. G. Prentiss, J. Opt. Soc. Am. B 5, 1613 (1988).

[9] M. D. Lukin and A. Imamoğlu, Phys. Rev. Lett. 84, 1419 (2000).

[10] M. D. Lukin, S. F. Yelin, and M. Fleischhauer, Phys. Rev. Lett. 84, 4232 (2000).

[11] M. Fleischhauer, U. Rathe, and M. O. Scully, Phys. Rev. A 46, 5856 (1992).

[12] G. S. Agarwal, Phys. Rev. Lett. 71, 1351 (1993).

[13] M. Jain, Phys. Rev. A 50, 1899 (1994).

[14] L. Hilico et al., Phys. Rev. A 46, 4397 (1992).

[15] J.-M. Courty, and S. Reynaud, Phys. Rev. A 46, 2766 (1992).

[16] This approximation is quite common. See, for example, ref. 17, pg. 410, Fig. 2.

[17] C. Cohen-Tannoudji, J. Dupont-Roc, G. Grynberg, Atom - Photon Interactions (John Wiley \& Sons, Inc, 1992).

[18] Details will be presented elsewhere.

[19] D. F. Walls and G. J. Milburn, Quantum Optics (Springer-Verlag, 1995).

[20] M. J. Collett and D. F. Walls, Phys. Rev. A 32, 2887 (1985).

[21] See, for example, H.-A. Bachor, A Guide to Experiments in Quantum Optics (John Wiley \& Sons, 1998).

[22] B. Julsgaard, A. Kozhekin, and E. S. Polzik, Nature (London) 413, 400 (2001).

[23] C. L. Garrido Alzar, M. França Santos, and P. Nussenzveig, in preparation. 